\title{
Hydromagnetic rotating flow of Casson fluid in Darcy- Forchheimer porous medium
}

\author{
Gauri Shanker Seth ${ }^{1}$, and Prashanta Kumar Mandal ${ }^{1, *}$ \\ 1 Department of Applied Mathematics, Indian Institute of Technology (Indian School of Mines), Dhanbad-826004, India
}

\begin{abstract}
Present study investigates three dimensional rotating flow of Casson fluid in the presence of magnetic field over a convectively heated linear stretching sheet. Concept of nonlinear radiative heat transfer is considered. The governing nonlinear partial differential equations are converted into ordinary differential equations with the help of similarity transformation and then solved by using shooting method along with Runge-Kutta-Fehlberg integration technique. The primary and secondary velocities and temperature profiles are plotted and analysed corresponding to various pertinent flow parameters. Also, the skin friction for both directions and rate of heat transfer at the surface are computed and explained.
\end{abstract}

\section{Introduction}

The effects of thermal radiation have massive impacts in industrial and engineering fields where devices are working at very high temperature under various nonisothermal conditions. Heat transfer via radiation is frequently applied in nuclear power plants, gas turbines, missiles and supersonic planes. Result for both small and large differences between fluid temperature at the sheet and free stream temperature can be obtained by considering nonlinear Rosseland diffusion approximation. The idea of nonlinear radiative heat transfer is presented by many researchers $[1,2]$.

Almost all industrial fluids disobey Newtonian law of viscosity. Therefore, study of non-Newtonian fluid flow is of supreme importance due to their inevitable presence in petrochemical industries, food and beverage processing and biotechnology. Casson fluid is one of the non-Newtonian fluid whose characteristics incluide human blood, jelly, honey, fruit juice with fibres etc. This type of fluid may find applications in medical and engineering sciences. Keeping in view the application of such flow, many researchers studied the flow of Casson fluid considering different aspects of the problem [3-6].

Rotating flow plays vital role in geophysics, astrophysics and fluid engineering. The engineering applications of rotating flows consist of filtration process based on centrifugal force, turbines, material processing and rotatary MHD generators. Wang [7] analysed viscous fluid flow near stretching surface in a rotating environment. Several researchers investigated the characteristics of flow behaviour considering rotation [8-10].

Flow of fluid through porous medium finds many applications in hydrology, agriculture, civil and petroleum engineering. In most of the cases, modeling and analysis of flow through porous medium are based on classical Darcy's theory, which is only valid for smaller porosity and lower velocity. Thus, at higher flow rates and higher permeability porous media, there is a departure from classical Darcy's law and in such a case inertial effect becomes important. Forchheimer [11] analysed such effects through square velocity vector in addition to Darcian velocity in momentum equation. A lot of researchers studied flow through porous medium considering Darcy-Forchheimer model [12-14].

Present investigation deals with the study of impact of rotation and non-linear thermal radiation over hydromagnetic flow of Casson fluid near a stretching sheet.

\section{Modeling of the problem}

Hydromagnetic Casson fluid flow generated by a linearly stretched sheet in Darcy-Forchheimer porous medium is considered. The sheet is aligned with the $x y$ plane. The stretching velocity of the surface in the $x$ direction is $u_{w}=a x$, where $a(>0)$ is the stretching rate. Fluid and sheet rotate in unison with uniform angular velocity $\Omega$ about $z$-axis in which direction magnetic field of uniform strength $B_{0}$ is applied. Schematic diagram of the problem is presented in Fig. 1.

The conservation of mass, momentum and energy equations (using Rosseland approximations for nonlinear thermal radiation) in a rotating frame are given by

$$
\frac{\partial u}{\partial x}+\frac{\partial v}{\partial y}+\frac{\partial w}{\partial z}=0
$$

*Corresponding author: ism.prashanta@gmail.com 


$$
\begin{gathered}
u \frac{\partial u}{\partial x}+v \frac{\partial u}{\partial y}+w \frac{\partial u}{\partial z}-2 \Omega v=v\{1+(1 / \beta)\} \frac{\partial^{2} u}{\partial z^{2}}-\frac{\sigma}{\rho} B_{0}{ }^{2} u \\
-\{1+(1 / \beta)\} \frac{v}{K^{*}} u-\left(C_{b} / \sqrt{K^{*}}\right) u^{2}, \\
u \frac{\partial v}{\partial x}+v \frac{\partial v}{\partial y}+w \frac{\partial v}{\partial z}+2 \Omega u=v\{1+(1 / \beta)\} \frac{\partial^{2} v}{\partial z^{2}}-\frac{\sigma}{\rho} B_{0}{ }^{2} v \\
-\{1+(1 / \beta)\} \frac{v}{K^{*}} v-\left(C_{b} / \sqrt{K^{*}}\right) v^{2}, \\
u \frac{\partial T}{\partial x}+v \frac{\partial T}{\partial y}+w \frac{\partial T}{\partial z}=\frac{\partial}{\partial z}\left[\left(\alpha+\frac{16 \sigma^{*} T^{3}}{3\left(\rho C_{p}\right) k^{*}}\right) \frac{\partial T}{\partial z}\right]
\end{gathered}
$$

The booundary conditions to be satisfied are given by

$$
\begin{gathered}
u=u_{w}=a x, v=0, w=0,-k \frac{\partial T}{\partial z}=h_{f}\left(T_{f}-T\right) \\
\text { at } \quad z=0, \\
u \rightarrow 0, v \rightarrow 0, T \rightarrow T_{\infty} \quad \text { as } z \rightarrow \infty,
\end{gathered}
$$

where,

$u, v, w, \rho, v, \sigma, \beta, C_{b}, K^{*}, T, T_{f}, T_{\infty}, \alpha, \sigma^{*}, k, C_{p}, k^{*}$ and $h_{f}$ are, respectively, the components of velocity along the directions of $x, y, z$ - axis, fluid density, kinematic coefficient of viscosity, electrical conductivity, Casson parameter, the drag coefficient, permeability of the porous medium, fluid temperature, temperature of the opposite side of the sheet, free stream temperature, thermal diffusivity, Stefan-Boltzmann constant, thermal conductivity of the fluid, specific heat at constant pressure, mean absorption coefficient and coefficient of heat transfer.

Now, we use the following similarity transformations :

$$
\left.\begin{array}{l}
\eta=\sqrt{(a / v)} z, \quad u=a x f^{\prime}(\eta), \quad w=-\sqrt{a v} f(\eta), \\
v=\operatorname{axg}(\eta), \theta(\eta)=\left(T-T_{\infty}\right) /\left(T_{f}-T_{\infty}\right) .
\end{array}\right\}
$$

With the help of eq. (6), eqs. (2) to (4) become

$$
\begin{aligned}
\{1+(1 / \beta)\} f^{\prime \prime \prime}+f f^{\prime \prime}+2 \lambda g- & {\left[M^{2}+K\{1+(1 / \beta)\}\right] f^{\prime} } \\
& -\left(1+F_{r}\right) f^{\prime 2}=0 \\
\{1+(1 / \beta)\} g^{\prime \prime}-2 \lambda f^{\prime}-[ & \\
+ & \left(M^{2}+K\{1+(1 / \beta)\}\right] g \\
\left(\left(1+R d\left(1+\left(\theta_{w}-1\right) \theta\right)^{3}\right) \theta^{\prime}\right)^{\prime}+\operatorname{Pr} f \theta^{\prime}=0 &
\end{aligned}
$$

Non-dimensional form of eqs. (5a) and (5b) are

$$
\left.\begin{array}{l}
f(\eta)=0, g(\eta)=0, f^{\prime}(\eta)=1, \\
\theta^{\prime}(\eta)=-\gamma\{1-\theta(\eta)\} \\
g(\eta) \rightarrow 0, f^{\prime}(\eta) \rightarrow 0, \theta(\eta) \rightarrow 0 \quad \text { as } \eta \rightarrow \infty,
\end{array}\right\}
$$

where, $\quad \lambda=\Omega / a, M^{2}=\left(\sigma B_{0}^{2}\right) /(\rho a), K=v /\left(a K^{*}\right)$,

$$
\operatorname{Pr}=\left(\rho v C_{p}\right) / k, F_{r}=\left(C_{b} x\right) /\left(\sqrt{K^{*}}\right), \theta_{w}=T_{f} / T_{\infty}(>1),
$$

$R d=\left(16 \sigma^{*} T_{\infty}^{3}\right) /\left(3 k k^{*}\right), \gamma=\left(h_{f} / k\right) \sqrt{(v / a)}$ are rotation parameter, magnetic parameter, porosity parameter, Prandtl number, local inertia coefficient, temperature ratio parameter, radiation parameter and Biot number.

In view of engineering and practical importance, significant physical entities of flow-field and temperature field are presented in terms of skin friction coefficients $C_{f x}, C_{f y}$ and Nusselt number $N u_{x}$ as

$$
\begin{aligned}
& \sqrt{\operatorname{Re}_{x}} C_{f x}=\{1+(1 / \beta)\} f^{\prime \prime}(0), \\
& \sqrt{\operatorname{Re}_{x}} C_{f y}=\{1+(1 / \beta)\} g^{\prime}(0), \\
& N u_{x} / \sqrt{\operatorname{Re}_{x}}=-\left[1+R d\left\{1+\left(\theta_{w}-1\right) \theta(0)\right\}^{3}\right] \theta^{\prime}(0),
\end{aligned}
$$

in which $\operatorname{Re}_{x}=u_{w} x / v$ is the local Reynolds number.

\section{Validation of numerical solution}

Eqs. (7) to (9) are coupled non-linear differential equations which are numerically solved by making use of shooting technique via Runge-Kutta-Fehlberg method. Suitable finite value of $\eta \rightarrow \infty$ say $\eta_{\infty}$ is taken as $\eta_{\infty} \approx 10$. The step size $\Delta \eta=0.001$ is issued to obtain the numerical solution. Suitable guesses values of $f^{\prime \prime}(0), \quad g^{\prime}(0)$ and $\theta^{\prime}(0)$ are choosen and the integration is carried out. Our results for $-f^{\prime \prime}(0)$ and $-g^{\prime}(0)$ agree excellently with the numerical results presented in $[7,8]$ (see Table 1).

\section{Discussion of results}

Obtained numerical solution of fluid velocities along $x$ and $y$-directions and fluid temperature profiles are plotted in Figs. 2 to 6. Default values for parameters are $M^{2}=\lambda=\beta=\gamma=0.5, K=0.2, F_{r}=0.1, R d=2, \theta_{w}=1.5$ and $\operatorname{Pr}=6.2$ unless otherwise stated.

It is evident from Figs. 2 and 3 that both primary velocity $f^{\prime}$ and secondary velocity $g$ decrease on increasing $M^{2} . f^{\prime}$ decreases on increasing $\lambda$ whereas $g$ increases near the sheet with greater values of $\lambda$. This means that magnetic field has a propensity to reduce the velocity of the fluid in both directions. Also, it is concluded that rotation reduces the primary flow and accelerates the secondary flow near the sheet which is in agreement with the characteristics of Coriolis force [15]. It is evident from Figs. 4 and 5 that both $f^{\prime}$ and $g$ decrease on incresing $K$ and $F_{r}, f^{\prime}$ decreases whereas $g$ increases as $\beta$ enlarges. Thus, porosity of the medium including inertia force has a tendency to reduce the primary and secondary fluid velocities. Casson parameter has opposite effects on primary and secondary 
velocities. Fig. 6 revels that, by increasing the values of of $R d, \theta_{w}$ and $\gamma$, an enhancement in the temperature field $\theta$ is observed. Also, the curves become $S$ - shaped for large values of temperature ratio. Increasing values of $\theta_{w}$ indicates larger surface temperature on opposite side of sheet compared to the free stream temperature.

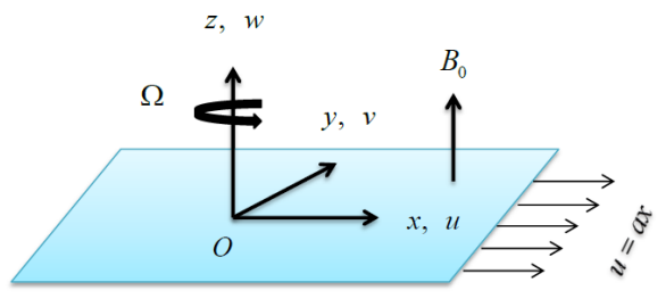

Fig. 1. Schematic diagram of the problem.

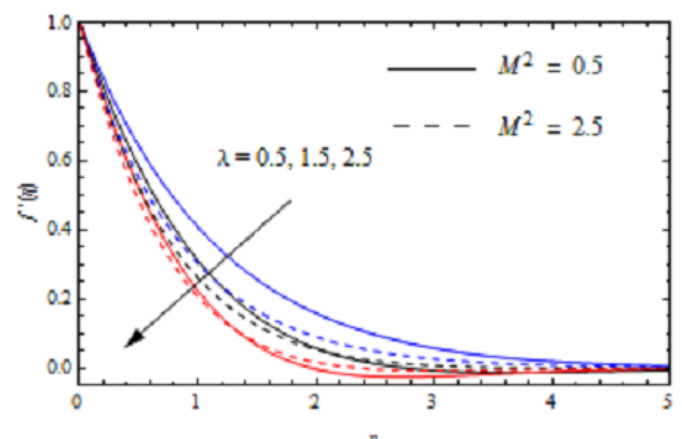

Fig. 2. Primary velocity profiles for different values of $M^{2}$ and $\lambda$.

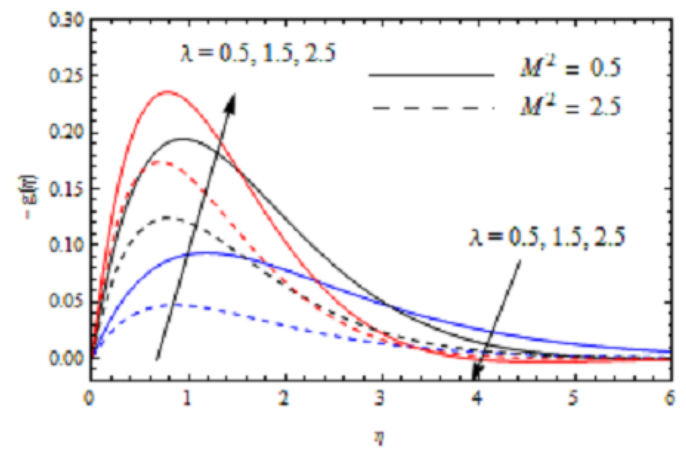

Fig. 3. Secondary velocity profiles for different values of $M^{2}$ and $\lambda$.

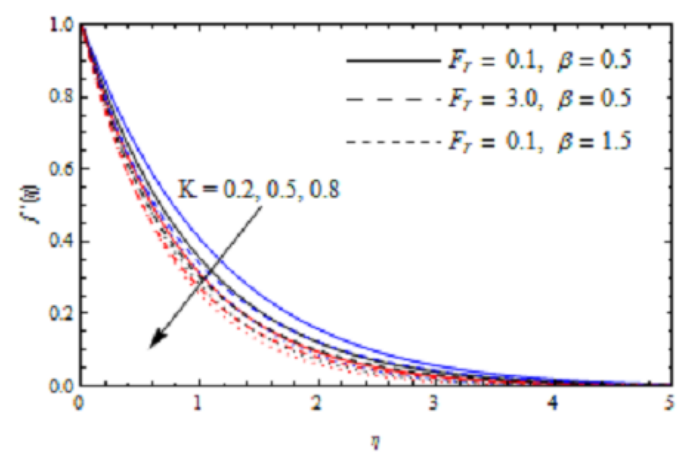

Fig. 4. Primary velocity profiles for different values of $K, F_{r}$ and $\beta$.

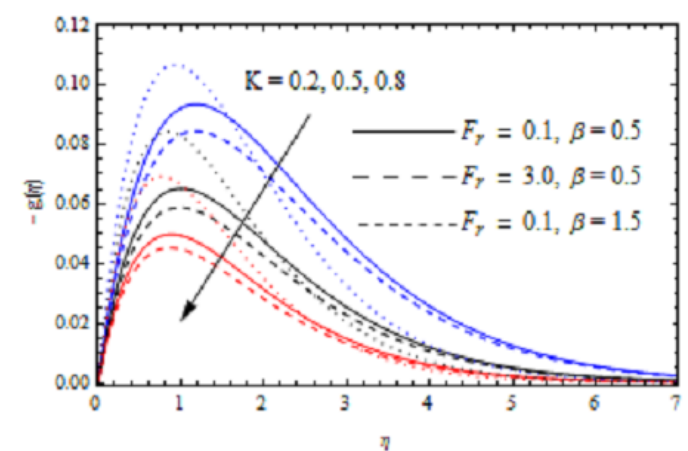

Fig. 5. Secondary velocity profiles for different values of $K, F_{r}$ and $\beta$.

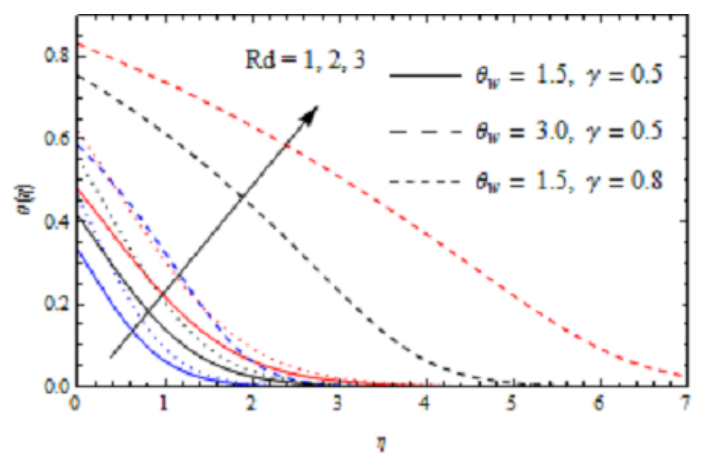

Fig. 6. Temperature profiles for different values of $R d$,

$\theta_{w}$ and $\gamma$.

Moreover, larger Biot number $\gamma$ produces stronger convection which leads to higher temperature field and more thermal boundary layer thickness.

Numerical values of skin friction coefficients due to primary and secondary flows and local Nusselt number, are presented in Tables 2 and 3. It is noticed that, $\sqrt{\operatorname{Re}_{x}} C_{f x}$ enhances with increasing $M^{2}, K, \lambda$ and $F_{r}$ and ruduces on increasing $\beta$, whereas there is an enhancement in $\sqrt{\operatorname{Re}_{x}} C_{f y}$ due to increasing $\lambda$ and reduction due to increament in $\beta, M^{2}, K$ and $F_{r}$. Due to rising values of $R d, \theta_{w}$ and $\gamma$, there is a marked enhancement in the values of $N u_{x} / \sqrt{\mathrm{Re}_{x}}$ whereas there is significant reduction in the values of $N u_{x} / \sqrt{\mathrm{Re}_{x}}$ due to the increment in $\lambda, M^{2}, \beta, K$ and $F_{r}$.

\section{Conclusion}

The important findings of this investigation are summarised below :

- The impact of rotation and Casson parameter on primary velocity is opposite to that of secondary velocity near the stretching sheet.

- Thermal radiation, temperatute ratio and Biot number enhance the heat transfer rate whereas it attenuates when magnetic field, rotation, Casson parameter, porosity of the medium and local inertia coefficient are intesified. 
Table 1. Comparison of present results with previous published results when $M^{2}=K=F_{r}=0$ and $\beta \rightarrow \infty$.

\begin{tabular}{|c|c|c|c|c|c|c|}
\hline \multirow{2}{*}{$\lambda$} & \multicolumn{2}{|c|}{ Wang [7] } & \multicolumn{2}{c|}{ Nazar et al. [8] } & \multicolumn{2}{c|}{ Present Results } \\
\cline { 2 - 7 } & $-f^{\prime \prime}(0)$ & $-g^{\prime}(0)$ & $-f^{\prime \prime}(0)$ & $-g^{\prime}(0)$ & $-f^{\prime \prime}(0)$ & $-g^{\prime}(0)$ \\
\hline 0 & 1 & 0 & 1 & 0 & 1 & 0 \\
\hline 0.5 & 1.1384 & 0.5128 & 1.1384 & 0.5128 & 1.13838096 & 0.51276039 \\
\hline 1 & 1.3250 & 0.8371 & 1.3250 & 0.8371 & 1.32502883 & 0.83709841 \\
\hline 2 & 1.6523 & 1.2873 & 1.6523 & 1.2873 & 1.65235799 & 1.28725883 \\
\hline
\end{tabular}

Table 2. Numerical data of skin friction coefficients and local Nusselt number.

\begin{tabular}{|c|c|c|c|c|c|c|c|}
\hline$M^{2}$ & $\lambda$ & $\beta$ & $K$ & $F_{r}$ & $-\sqrt{\operatorname{Re}_{x}} C_{f x}$ & $-\sqrt{\operatorname{Re}_{x}} C_{f y}$ & $N u_{x} / \sqrt{\operatorname{Re}_{x}}$ \\
\hline 0.5 & 0.5 & 0.5 & 0.2 & 0.1 & 2.62934 & 0.626940 & 1.32361 \\
\hline 1.5 & \multirow[b]{2}{*}{0.5} & \multirow[b]{2}{*}{0.5} & \multirow[b]{2}{*}{0.2} & \multirow[b]{2}{*}{0.1} & 3.12564 & 0.511611 & 1.31136 \\
\hline 2.5 & & & & & 3.56354 & 0.441663 & 1.29937 \\
\hline \multirow[b]{2}{*}{0.5} & 1.5 & \multirow[b]{2}{*}{0.5} & \multirow[b]{2}{*}{0.2} & \multirow[b]{2}{*}{0.1} & 3.02305 & 1.592230 & 1.30437 \\
\hline & 2.5 & & & & 3.44740 & 2.286630 & 1.27785 \\
\hline \multirow[b]{2}{*}{0.5} & \multirow[b]{2}{*}{0.5} & 1.0 & \multirow[b]{2}{*}{0.2} & \multirow[b]{2}{*}{0.1} & 2.05930 & 0.538751 & 1.31107 \\
\hline & & 1.5 & & & 1.85281 & 0.500770 & 1.30326 \\
\hline \multirow[b]{2}{*}{0.5} & \multirow[b]{2}{*}{0.5} & \multirow[b]{2}{*}{0.5} & 0.5 & \multirow[b]{2}{*}{0.1} & 3.07888 & 0.520507 & 1.31257 \\
\hline & & & 0.8 & & 3.47993 & 0.453420 & 1.30174 \\
\hline \multirow[b]{2}{*}{0.5} & \multirow[b]{2}{*}{0.5} & \multirow[b]{2}{*}{0.5} & \multirow[b]{2}{*}{0.2} & 1 & 3.10462 & 0.602219 & 1.31801 \\
\hline & & & & 3 & 3.54763 & 0.581886 & 1.30691 \\
\hline
\end{tabular}

Table 3. Numerical data of local Nusselt number.

\begin{tabular}{|c|c|c|c|}
\hline$R d$ & $\theta_{w}$ & $\gamma$ & $N u_{x} / \sqrt{\mathrm{Re}_{x}}$ \\
\hline 1 & 1.5 & 0.5 & 0.86382 \\
\hline 2 & \multirow[b]{2}{*}{1.5} & \multirow[b]{2}{*}{0.5} & 1.32361 \\
\hline 3 & & & 1.75035 \\
\hline \multirow[b]{2}{*}{2} & 2 & \multirow[b]{2}{*}{0.5} & 1.92839 \\
\hline & 3 & & 4.00365 \\
\hline \multirow[b]{2}{*}{2} & \multirow[b]{2}{*}{1.5} & 0.2 & 0.58673 \\
\hline & & 0.8 & 1.84113 \\
\hline
\end{tabular}

- The behaviour of magnetic field, porosity of the medium and local inertia coefficient on primary and secondary velocities are same.

- Rotation and porosity of the medium have same effect on skin friction coefficient in both the directions.

- For sufficiently large temperature ratio, the temperature profiles become S - shaped which indicates the occurrence of adiabatic case.

\section{References}

1. R. Cortell, J. King Saud Univ-Sci., 26, 2, 161167 (2014).

2. A. Mushtaq, M. Mustafa, T. Hayat, A. Alsaedi, J. Taiwan Institute of Chem. Eng., 45, 11761183 (2014).

3. S. Nadeem, R. U. Haq, N. S. Akbar, IEEE Trans. Nanotech., 13, 109-115 (2014).

4. S. A. Shehzad, T. Hayat, A. Alsaedi, J. Appl. Fluid Mech., 9, 1, 215-223 (2016).
5. C. Sulachana, G. P. Aswinkumar, N. Sandeep, J. Niger. Math. Soc., 35, 1. 128-141 (2016).

6. I. S. Oyelakin, S. Mondal, P. Sibanda, Alexandria Eng. J., 55, 1025-1035 (2016).

7. C. Y. Wang, Z. Angew Math. Phys (ZAMP), 39, 177-185 (1988).

8. R. Nazar, N. Amin, I. Pop, Mech. Res. Commun., 31, 121-128 (2004).

9. M. Sheikholeslami, M. Hatami, D. D. Ganji, J. Mol. Liq., 190, 112-120 (2014).

10. M. Mustafa, A. Mushtaq, T. Hayat, A. Alsaedi, Plos One, 11, 2, 1-16 (2016).

11. P. Forchheimer, Zeitschrift Ver D. Ing., 45, 1782-1788 (1901).

12. M. A. Seddeek, J. Colloid Interface Sci., 293, 137-142 (2006).

13. B. K. Jha, Appl. Math., 2, 1432-1436 (2011).

14. M. A. Sadiq, T. Hayat, Results Phys., 6, 884890 (2016).

15. H. P. Greenspan, The Theory of Rotating Fluids (Cambridge Univ. Press, New York, 1968). 